Mol Reprod Dev. 2018 October ; 85(10): 802-804. doi:10.1002/mrd.23053.

\title{
Prps111, a Testis-specific Gene, Is Dispensable for Mouse Spermatogenesis
}

\author{
Zhuqing Wang ${ }^{1}$, Sandy Lee ${ }^{1}$, Daniel Oliver ${ }^{1}$, Shuiqiao Yuan ${ }^{1}$, Chong Tang $^{1}$, Yue Wang ${ }^{1}$, \\ Huili Zheng ${ }^{1}$, and Wei Yan ${ }^{1,2,3,{ }^{*}}$ \\ ${ }^{1}$ Department of Physiology and Cell Biology, University of Nevada, Reno School of Medicine, \\ Reno, NV 89557 \\ ${ }^{2}$ Department of Obsterics and Gynecology, University of Nevada, Reno School of Medicine, \\ Reno, NV 89557 \\ ${ }^{3}$ Department of Biology, University of Nevada, Reno, Reno, NV 89557
}

\section{Keywords \\ Prps 111; spermatogenesis; CRISPR/Cas9}

\begin{abstract}
Phosphoribosyl pyrophosphate synthetase (PRPS) plays an important role in nucleotide biosynthesis by transferring pyrophosphate groups from adenosine triphosphate (ATP) to ribose-5-phophate to form 5-phosphoribosyl-1-pyrophosphate (PRPP)(Cunningham et al. 2014; de Brouwer et al. 2010). The PRPS family contains three members encoded by Prps1, Prps2 and Prps111 (de Brouwer et al. 2010; Taira et al. 1989). Prps1 and Prps2 are X-linked genes expressed in a wide variety of organs/tissues, whereas Prps111 on chromosome 12 is exclusively expressed in the testis (Taira et al. 1989). Despite its testis-specific expression, the function of Prps111 remains unknown.
\end{abstract}

To explore the physiological role of Prps111, we examined its expression profile and generated a global Prps111 knockout (KO) mouse line. Prps111 and Prps1, but not Prps2, were abundantly expressed in the testis, and unlike Prps 1 which was also expressed in other organs, Prps111 expression appeared to be restricted to the testis (Fig. 1A). The onset of Prps111 mRNA expression was at postnatal day 21 (P21) and its levels remained high thereafter (Fig. 1B), suggesting that Prps 111 is mainly expressed in haploid male germ cells, i.e., round and elongating/elongated spermatids, in adult murine testes. In contrast, levels of Prps 1 mRNAs were lower prior to P7 and became much higher thereafter (Fig. 1B), suggesting that it is expressed in all types of spermatogenic cells in adult murine testes. The cellular origin of Prps 1 and Prps 111 mRNA expression was further confirmed by qPCR analyses using pachytene spermatocytes, round and elongating spermatids purified from the adult murine testes (Fig. 1C). Next, we generated Prps111 global knockout (KO) mice using

\footnotetext{
*Corresponding author. wyan@med.unr.edu.

Please see Supplemental Materials for methods and primer sequences used in this study.

CONFLICT OF INTEREST

The authors declare no conflict of interest.
} 
the CRISPR/Cas9-based genome editing technology. A PCR-based genotyping method was developed such that presence of external and absence of internal PCR products would indicate homozygous Prps111 KO (Fig. 1D and Supplemental Table S1). In addition to genotyping assays, the complete lack of Prps 111 mRNAs in the testes of Prps $111 \mathrm{KO}$ males was further confirmed using qPCR analyses (Fig. 1E).

A 3-month-long fertility test showed that Prps 111 KO males had normal fertility (data not shown). Testicular weight and histology of adult Prps111 KO males were comparable to those of WT males (Fig. 1F and Fig. 1G). Prps111 KO males also displayed normal sperm counts and motility, as well as sperm morphology (Fig.1H and Fig. 1I). Levels of Prps1 and Prps 2 mRNAs were slightly, although not significantly, increased in Prps $111 \mathrm{KO}$ compared to WT testes (Fig. 1E). No significant upregulation of either Ppat or Ahcy, two genes known to encode proteins that can bypass PRPP (de Brouwer et al. 2010), was observed either (Fig. 1E). Taken together, our data suggest that, despite its testis-exclusive expression, Prps111 is dispensable for spermatogenesis in mice.

\section{Supplementary Material}

Refer to Web version on PubMed Central for supplementary material.

\section{ACKNOWLEDGMENTS}

This work was supported by grants from the NIH (HD071736, HD085506 and P30GM110767 to WY) and the Templeton Foundation (PID: 50183 to WY).

Grant sponsor: National Institutes of Health (NIH) and the Templeton Foundation

Grant number: HD071736, HD085506, P30GM110767, and 50183

\section{REFERENCES}

Cunningham JT, Moreno MV, Lodi A, Ronen SM, Ruggero D. 2014 Protein and nucleotide biosynthesis are coupled by a single rate-limiting enzyme, PRPS2, to drive cancer. Cell 157(5): 1088-1103. [PubMed: 24855946]

de Brouwer AP, van Bokhoven H, Nabuurs SB, Arts WF, Christodoulou J, Duley J. 2010 PRPS1 mutations: four distinct syndromes and potential treatment. Am J Hum Genet 86(4):506-518. [PubMed: 20380929]

Taira M, Iizasa T, Yamada K, Shimada H, Tatibana M. 1989 Tissue-differential expression of two distinct genes for phosphoribosyl pyrophosphate synthetase and existence of the testis-specific transcript. Biochimica et biophysica acta 1007(2):203-208. [PubMed: 2537655] 

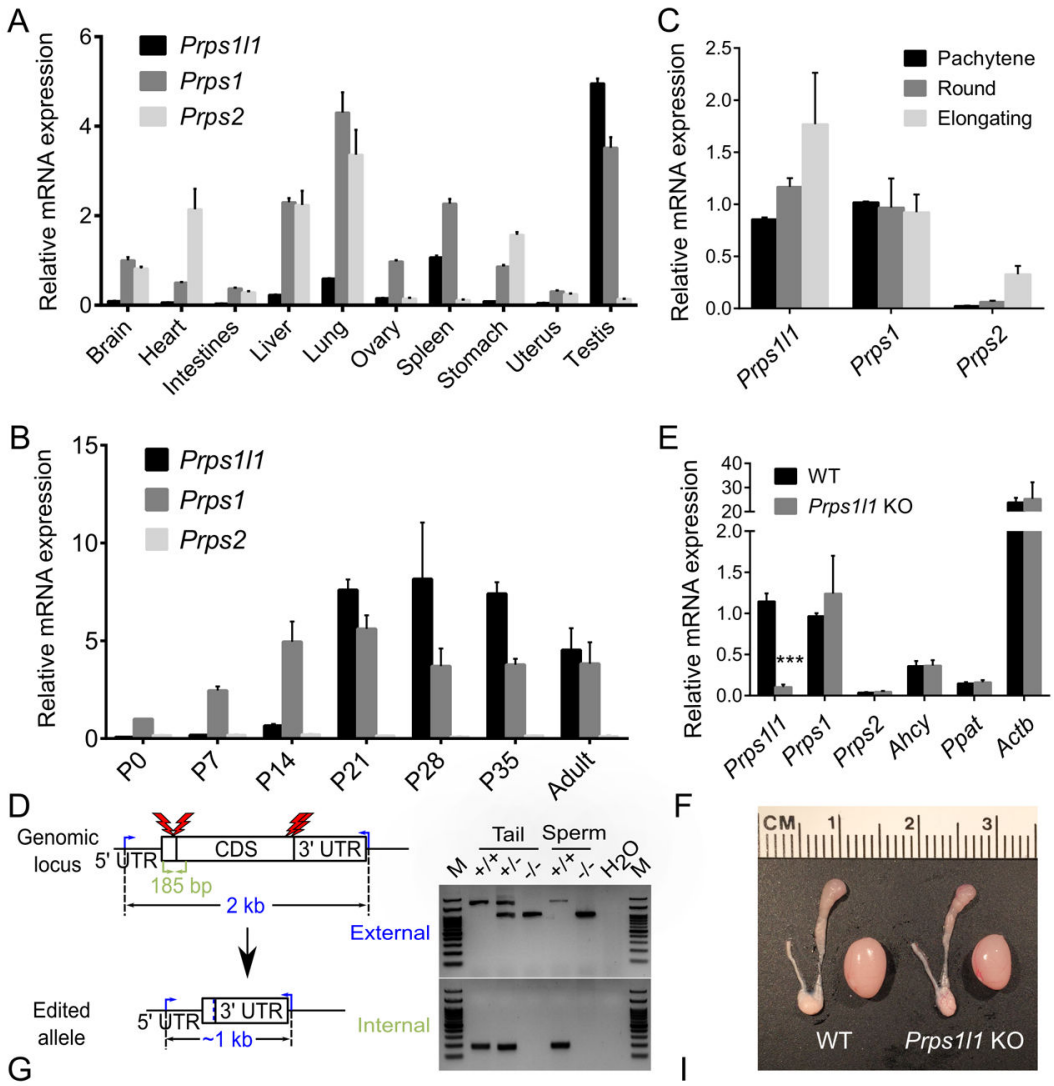

G

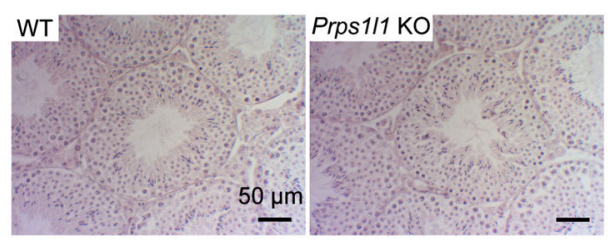

$\mathrm{H}$

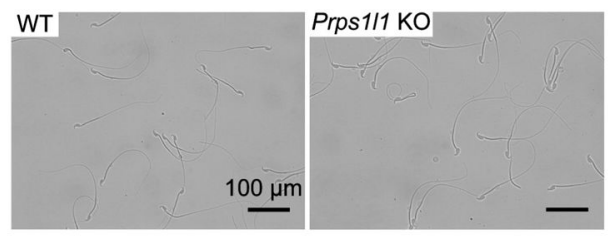

E
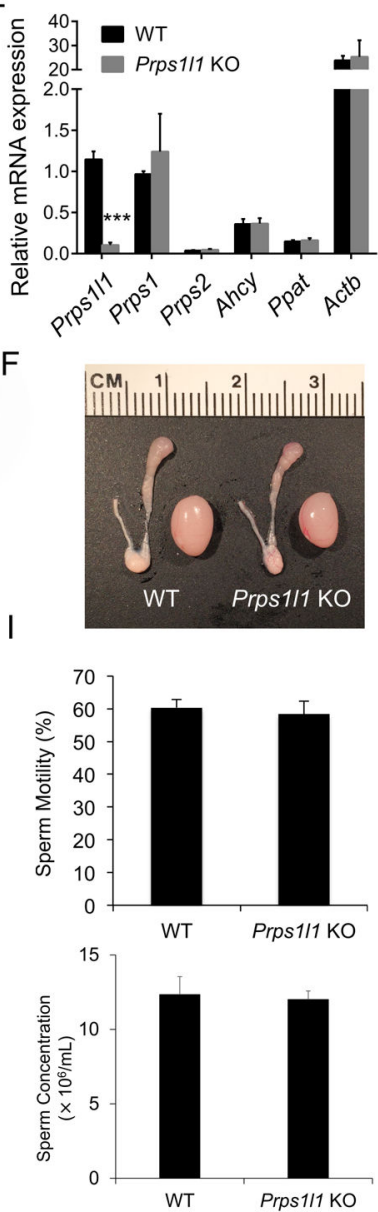

Figure 1.

Prps111 is dispensable for spermatogenesis despite its high expression in the testis. A. qPCR analyses of mRNA expression levels of three PRPS genes (Prps1, Prps2 and Prps111) in ten organs of adult mice. Expression levels were normalized to Gapdh. Data are presented as means \pm SEM, $n=3$. B. qPCR analyses of mRNA expression levels of three PRPS genes (Prps1, Prps2 and Prps111) in developing murine testes at postnatal day 0 (P0), P7, P14, P21, P28, P35 and adulthood. Expression levels were normalized to Gapdh. Data are presented as means \pm SEM, n=3. C. qPCR analyses of Prps 111 mRNA levels in pachytene spermatocytes, round and elongating spermatids purified from WT adult murine testes. Expression levels were normalized to Gapdh. Data are presented as means \pm SEM, n=3. D. Schematic 
illustration of the generation of Prps111 KO mouse and a representative genotyping result of Prps111 heterozygous (+/-), homozygous (-/-) and wild type (+/+). M, marker. The red lightning bolt represents gRNAs used, and its right and left orientations indicate the gRNAs targeting the reverse and forward strands of the genomic DNA, respectively. Blue arrows show the position of external primers, while light green arrows indicate that of internal primers. The expected size of PCR products is indicated in the same color. E. qPCR analyses of mRNA expression levels of the three PRPS genes (Prps1, Prps 2 and Prps111) and two related genes (Ppat and Ahcy) in Prps111 KO testes. Expression levels were normalized to Gapdh. Data are presented as means \pm SEM, n $=3, * * * \mathrm{p}<0.001$. F. Normal morphology of WT and Prps $111 \mathrm{KO}$ testes. One unit on the ruler is $1 \mathrm{~mm}$. G. A representative image of Haematoxylin and Eosin (HE)-stained WT and Prps111 KO testes section. Scale bar=50 $\mu \mathrm{m}$. H. A representative phase-contrast micrograph showing normal morphology of WT and Prps 111 KO sperm. Scale bar $=100 \mu \mathrm{m}$. I. Sperm motility and concentration assays on adult Prps111 KO males. 